\title{
FUNCTIONALS WITH VALUES IN THE NON-ARCHIMEDEAN FIELD OF LAURENT SERIES AND THEIR APPLICATIONS TO THE EQUATIONS OF ELASTICITY THEORY. I ${ }^{1}$
}

\author{
M. RADYNA \\ Department of Informatics Belorussian State University of Informatics and \\ Radioelectronics \\ P. Brovki 6, Minsk, 220013, Belarus \\ E-mail: kolya@im.bas-net.by
}

Received February 5, 2002

\begin{abstract}
Functionals with values in Non-Archimedean field of Laurent series applied to the definition of generalized solution (in the form of soliton) of the Hopf equation. Calculation method for the profile of infinitely narrow soliton is proposed. Applying this method, calculations of profiles are reduced to the nonlinear system of algebraic equations in $\mathbf{R}^{n+1}, n>1$. It is shown that there is a possibility to find out some of the solutions of this system using the Newton iteration method. Example and numerical test are considered.
\end{abstract}

Key words: generalized functions, distributions, conservation law, Hopf equation, soliton, shock waves.

\section{INTRODUCTION}

While working at Los Alamos in 1943-44, von Neumann became convinced that the calculation of the flows of compressible fluids containing strong shocks could be accomplished only by numerical methods. He conceived the idea of capturing shocks, i.e., of ignoring the presence of a discontinuity. Employing a Lagrangian description of compressible flow, setting heat conduction and viscosity equal to zero, von Neumann replaced space and time derivatives by

\footnotetext{
${ }^{1}$ Dedicated to Tatsiana Radyna
} 
symmetric difference quotients. Calculations using this scheme were carried out; the approximation resulting from these calculations (see [20]) showed oscillations on the mesh scale behind the shock. Von Neumann boldly conjectured that the oscillations in velocity represent the heat energy created by the irreversible action of the shock, and that as $\Delta x$ and $\Delta t$ tend to zero, the approximate solutions tend in the weak sense to the discontinuous solution of the equations of compressible flow.

In [14] it was counter-conjectured that von Neumann was wrong in his surmise, i.e., that although the approximate solutions constructed by his method do converge weakly, the weak limit fails to satisfy the law of conservation of energy.

In [8] J. Goodman and P. Lax investigated von Neumann's algorithm applied to the scalar equation

$$
u_{t}+u u_{x}=0
$$

(it is called the Hopf equation), in the semi-discrete case. Using numerical experimentation and analytical techniques they demonstrated the weak convergence of the oscillatory approximations, and that the weak limit fails to satisfy the scalar equation in question.

Von Neumann's dream of capturing shocks was realized in his joint work with Richtmyer in 1950, see [21]. Oscillations were eliminated by the judicious use of artificial viscosity; solutions constructed by this method converge uniformly except in a neighborhood of shocks, where they remain bounded and are spread out over a few mesh intervals. The limits appear to satisfy the conservation laws of compressible flow. The conservation of mass and momentum is the consequence of having approximated these equations by difference equations in conservation form; but the von Neumann-Richtmyer difference approximation to the energy equation is not in conservation form.

In the paper [10] T. Hou and P. Lax compared the results of a von Neumann-Richtmyer calculation with the weak limit of calculations performed by von Neumann's original method. In the paper [10] P. Lax asserts that the difference scheme of von Neumann, because of the centering of the difference quotients, is dispersive; it is this quality that is responsible for the oscillatory nature of the solutions.

E. Hopf [9] studied (1.1) and defined the generalized solution for this equation. He considered the perturbed equation

$$
u_{t}+u u_{x}=\mu u_{x x}, \quad \text { where } \mu \rightarrow 0
$$

which converges to the equation (1.1).

By a generalized solution $u$ of (1.1) or (1.2), $\mu=0 \mathrm{E}$. Hopf meant a function $u$ that is measurable and quadratically integrable in every closed rectangle in the open semiplane $t>0$ and that satisfies the relation

$$
\iint\left[u g_{t}+\frac{u^{2}}{2} g_{x}\right] d x d t=0,
$$


where $g$ is an arbitrary function of class $C^{1}$ in $t>0$ that vanishes outside some circle lying entirely in $t>0$. He assert: Every limit function $u$ obtained from the solution of (1.2) as $\mu \rightarrow+0$ is a generalized solution of (1.1). By a generalized solution $u$ of (1.2) E. Hopf meant a function $u$ that is measurable and quadratically integrable in every closed rectangle in the open semiplane $t>0$ and that satisfies the relation

$$
\iint\left[u f_{t}+\frac{u^{2}}{2} f_{x}+\mu u f_{x x}\right] d x d t=0
$$

where $f$ is an arbitrary function of class $C^{2}$ in $t>0$ that vanishes outside some circle lying entirely in $t>0$.

This method is called the "disappearing viscosity" method. It was developed by E. Hopf [9], O.A. Oleinik [23], P. Lax [10; 15].

There is also the "zero dispersion limit" method developed by P. Lax $[8 ; 14]$ V.P. Maslov and his collaborators [5; 18]. The idea is to use the following equation

$$
u_{t}+u u_{x}=\varepsilon^{2} u_{x x x}, \quad \varepsilon \rightarrow 0
$$

for (1.1). V.P. Maslov and his collaborators constructed an asymptotic solutions for (1.1). They speculated on the fact that equations (1.2) and (1.5) have solutions in the class $C^{\infty}$ functions. For example, a particular solution of the equation (1.5) is the function

$$
u(x, t)=c+4-12 \cosh ^{-2}\left(\frac{x-c t}{\varepsilon}\right), c>0,
$$

which represents so-called an infinitely narrow soliton. A particular solution of (1.2) will be, for instance,

$$
u(x, t)=2-2 \tanh \left(\frac{x-2 t}{\mu}\right),
$$

which converges to a discontinues function.

On the other hand, in the paper [30] S.L. Sobolev (1936) introduced mathematical basics of the theory of generalized functions, he introduced the idea of a generalized function as a functional and proposed the concept of generalized solutions of a linear differential equation. These generalized functions and generalized solutions were developed by L. Schwartz [29]. However, SobolevSchwartz distributions can not applied to nonlinear differential equations. For example, to substitute generalized function $H(x-v t$ ) (where $H$ is the Heaviside function) into the (1.1) one need to define the product of two distributions $H$ and $H^{\prime}$ for the term $u u_{x}$. However, in $1954 \mathrm{~L}$. Schwartz showed that it is impossible to introduce an associate multiplication in the space of distributions. 
Starting from 1982 in the works by J.-F. Colombeau [3], M. Oberguggenberger [22], H. Biagioni [2], E. Rozinger [27], A.Y. Le Roux [4], Yu. Egorov [7], J.-A. Marti [17], A. Delcroix, D.Scarpalézos [6], B. Keyfitz [12], A. Antonevich, Ya. Radyno [1], T. Todorov [31], S. Pilipović [19] and others, a new theory of generalized functions is developed. Such functions form the algebra and contain distributions.

In general, nonlinear generalized functions are classes of equivalent smooth functions. Clearly that, one should pay attention to this approach in order to consider nonlinear differential equations. However we are now in a position to develop a new point of view on generalized functions and their applications to nonlinear equations. Namely, it is necessary to use an integral nature of a conservation law. Conservation laws are integral expressions from physical point of view and it is natural to consider an integral form of conservation laws. Moreover, we want to develop new point of view on conservation laws using the concept of functionals with values in the Non-Archimedean field of Laurent series. We call such functionals as $\mathbf{R}\langle\varepsilon\rangle$-distributions [24; 26]. In addition, we give the definition of the special kind of solutions of the some conservation laws in the sense of $\mathbf{R}\langle\varepsilon\rangle$-distributions and consider the method for the numerical calculations of the smooth shocks and soliton like solutions of the Hopf equation and equations of elasticity theory in the mentioned sense. This method based on orthogonal system of the Hermite functions as a base for calculation of such solutions (i.e. shocks and infinitely narrow solitons). Calculations of profiles of infinitely narrow soliton and shock wave are reduced to the nonlinear system of algebraic equations in $\mathbf{R}^{n+1}, n>1$. We proved, using the Schauder fixed point theorem [28], that the mentioned system has at least one solution in $\mathbf{R}^{n+1}$. We showed that there is possibility to find out some of the solutions of this system using the Newton iteration method [11]. We considered examples and numerical tests. We also should emphasis that proposed numerical approach do not use a difference scheme.

First, let us consider a bit of theory which we will apply to conservation laws.

\section{NON-ARCHIMEDEAN FIELD OF LAURENT SERIES AND $\mathbf{R}\langle\varepsilon\rangle$-DISTRIBUTIONS}

The theory of Non-Archimedean fields was considered in the book by A.H. Lightstone and A. Robinson [16].

Definition 2.1. A Laurent series is a formal object

$$
\sum_{n=0}^{\infty} \xi_{n+k} \varepsilon^{n+k}
$$

where $k$ is a fixed (i.e., fixed for this Laurent series), each $\xi_{i} \in \mathbf{R}$, and either $\xi_{k} \neq 0$ or each $\xi_{i}=0$. 
The Laurent series $\sum_{n=0}^{\infty} \xi_{n} \varepsilon^{n}$, where $\xi_{0}=1$ and $\xi_{n}=0$ if $n>0$, is denoted by 1 . It is easy to see that the Laurent series is a field. Let us denote it by $\mathbf{R}\langle\varepsilon\rangle$. The norm on the field of Laurent series can define

$$
|x|_{\nu}=e^{-\nu(x)} \quad \text { for } \text { each } \quad x \in \mathbf{R}\langle\varepsilon\rangle
$$

(in place of $e$ one can use any number greater then 1). The function $\nu(x)$ is a Non-Archimedean valuation. Define $\nu(0)=\infty$,

$$
\nu\left(\sum_{n=0}^{\infty} \xi_{n+k} \varepsilon^{n+k}\right)=k \quad \text { if } \quad \sum_{n=0}^{\infty} \xi_{n+k} \varepsilon^{n+k} \neq 0, \quad \xi_{k} \neq 0
$$

The norm $|\cdot|_{\nu}$ have properties

1. $|x|_{\nu}=0$ if and only if $x=0$,

2. $|x y|_{\nu}=|x|_{\nu} \cdot|y|_{\nu}$,

3. $|x+y|_{\nu} \leq \max \left\{|x|_{\nu},|y|_{\nu}\right\}$.

Here, we propose a general construction of the $\mathbf{R}\langle\varepsilon\rangle$-valued generalized functions [25]. These objects are a natural generalization of Sobolev-Schwartz distributions. We call them as $\mathbf{R}\langle\varepsilon\rangle$-distributions.

1. Consider all functions $f(x, \varepsilon) \in C^{\infty}(\mathbf{R} \times(0,1))$ such that integrals

$$
\int_{-\infty}^{+\infty} f(x, \varepsilon) \psi(x) d x
$$

exist for any $\varepsilon$ and for all $\psi(x)$ from a given class of functions $\mathcal{X}$ (X can be $C_{0}^{\infty}(\mathbf{R}), \mathcal{S}(\mathbf{R})$ and etc. $)$.

2. Suppose also that $\int_{-\infty}^{+\infty} f(x, \varepsilon) \psi(x) d x$ is a number $a_{f, \varepsilon}(\psi)$ from the field of Laurent series $\mathbf{R}\langle\varepsilon\rangle$.

3. The two functions $f(x, \varepsilon)$ and $g(x, \varepsilon)$ call equivalent with respect to test functions $\mathcal{X}$ if and only if

$$
\int_{-\infty}^{+\infty} f(x, \varepsilon) \psi(x) d x=a_{f, \varepsilon}(\psi)=a_{g, \varepsilon}(\psi)=\int_{-\infty}^{+\infty} g(x, \varepsilon) \psi(x) d x
$$

The equality means in sense of the field of Laurent series $\mathbf{R}\langle\varepsilon\rangle$ for all functions $\psi \in \mathcal{X}$. Classes of equivalent functions call $\mathbf{R}\langle\varepsilon\rangle$-functions. The expression

$$
\int_{-\infty}^{+\infty} f(x, \varepsilon) \psi(x) d x
$$


associates a number from $\mathbf{R}\langle\varepsilon\rangle$ with every $\psi$. Such a quantity is called a functional. In this case a linear functional map $\mathcal{X}$ into the Non-Archimedean field $\mathbf{R}\langle\varepsilon\rangle$. Call these functionals as $\mathbf{R}\langle\varepsilon\rangle$-distributions.

Thus,

Proposition 2.1. $\mathbf{R}\langle\varepsilon\rangle$-function $f(x, \varepsilon)=0$ if and only if

$$
\int_{-\infty}^{+\infty} f(x, \varepsilon) \psi(x) d x=0 \in \mathbf{R}\langle\varepsilon\rangle
$$

for every $\psi$ from $\mathcal{X}$.

Let denote the set of all $\mathbf{R}\langle\varepsilon\rangle$ - distributions by $\mathcal{R}(\mathcal{X})$

Remark 2.1. Recall that the idea of representation of a function $f \in$ $L_{\text {loc }}^{1}(\mathbf{R})$ in terms of a linear functional

$$
C_{0}^{\infty}(\mathbf{R}) \ni \psi \vdash \int_{-\infty}^{+\infty} f(x) \psi(x) d x \in \mathbf{R}
$$

is based on well-known proposition that if $f \in L_{l o c}^{1}(\mathbf{R})$ and

$$
\int_{-\infty}^{+\infty} f(x) \psi(x) d x=0, \quad \forall \psi \in C_{0}^{\infty}(\mathbf{R})
$$

then $f=0$ almost everywhere.

Let us consider an example of the $\mathbf{R}\langle\varepsilon\rangle$-distribution.

Example 2.1. Take $\mathcal{X}=C_{0}^{\infty}(\mathbf{R})$ and $f(x, \varepsilon)=\varphi(x / \varepsilon), \varphi(x) \in C_{0}^{\infty}(\mathbf{R})$ then $\mathbf{R}\langle\varepsilon\rangle$-distribution can be written in the following form.

$$
\int_{-\infty}^{+\infty} \varphi(x / \varepsilon) \psi(x) d x=\varepsilon \int_{-\infty}^{+\infty} \varphi(x) d x \psi(0)+\varepsilon^{2} \int_{-\infty}^{+\infty} x \varphi(x) d x \frac{\psi^{\prime}(0)}{1 !}+\ldots
$$

Note that $\varphi(x / \varepsilon)$ converges to the function

$$
u(x)= \begin{cases}\varphi(0), & \text { if } x=0, \\ 0, & \text { if } x \neq 0 .\end{cases}
$$


The last function almost everywhere equals to zero.

Like Sobolev-Schwartz distributions we can differentiate $\mathbf{R}\langle\varepsilon\rangle$-distributions. For example,

$$
\begin{gathered}
\int_{-\infty}^{+\infty} \frac{d}{d x} \varphi(x / \varepsilon) \psi(x) d x=-\int_{-\infty}^{+\infty} \varphi(x / \varepsilon) \frac{d}{d x} \psi(x) d x \\
-\int_{-\infty}^{+\infty} \varphi(x / \varepsilon) \frac{d}{d x} \psi(x) d x=-\varepsilon \int_{-\infty}^{+\infty} \varphi(x) d x \psi^{\prime}(0)-\varepsilon^{2} \int_{-\infty}^{+\infty} x \varphi(x) d x \frac{\psi^{\prime \prime}(0)}{1 !}-\ldots
\end{gathered}
$$

It is evident that $\mathbf{R}\langle\varepsilon\rangle$-distributions are more general objects than Sobolev-Schwartz distributions [29;30].

\section{CONSERVATION LAWS:}

\section{NON-ARCHIMEDEAN APPROACH}

A conservation law asserts that the rate of change of the total amount of substance contained in a fixed domain $G$ is equal to the flux of that substance across the boundary of $G$. Denoting the density of that substance by $u$, and the flux by $f$, the conservation law is given by

$$
\frac{d}{d t} \int_{G} u(t, x) d x=-\int_{\partial G} f \vec{n} d S .
$$

Applying the divergence theorem and taking $d / d t$ under the integral sign we obtain

$$
\int_{G}\left(u_{t}+\operatorname{div} f\right) d x=0 .
$$

Dividing by vol $(G)$ and shrinking $G$ to a point where all partial derivatives of $u$ and $f$ are continuous we obtain the differential conservation law

$$
u_{t}(t, x)+\operatorname{div} f(u(t, x))=0 .
$$

Note, that if $f(u)=u^{2} / 2$ then we obtained the Hopf equation (1.1). In general, previous calculations are based on the following well known proposition.

Proposition 3.1. If $G \in L_{l o c}^{1}(\mathbf{R})$ and

$$
\int_{-\infty}^{+\infty} G(x) \psi(x) d x=0 \text { for } \forall \psi \in C_{0}^{\infty}(\mathbf{R})
$$

then $G=0$ almost everywhere. 
Definition 3.1. Let us consider two sets of the smooth functions, depending on a small parameter $\varepsilon \in(0,1]$. Let us take all functions $v(t, x, \varepsilon)$ which have the type

$$
v(t, x, \varepsilon)=l_{0}+\Delta l \varphi\left(\frac{x-c t}{\varepsilon}\right),
$$

$l_{0}, \Delta l, c$ are real numbers, $\Delta l \neq 0$ and $\varphi \in \mathcal{S}(\mathbf{R}), \int_{-\infty}^{+\infty} \varphi(y) d y=1$. We denote this set of functions by $I$ and call it as a set of infinetely narrow solitons.

Definition 3.2. Now, let us take all functions $w(t, x, \varepsilon)$ which have the form

$$
w(t, x, \varepsilon)=h_{0}+\Delta h H\left(\frac{x-a t}{\varepsilon}\right)
$$

$h_{0}, \Delta h, a$ are real numbers, $\Delta h \neq 0$,

$$
H(x)=\int_{-\infty}^{x} \theta(y) d y, \quad \int_{-\infty}^{+\infty} \theta(y) d y=1
$$

and $\theta \in \mathcal{S}(\mathbf{R})$. We denote this set of functions by $J$, and call it as a set of shock waves.

It is natural to consider conservation laws as an integral expressions which contain the time $t$ as parameter. Therefore, we introduce the following concept.

Definition 3.3. The function $v \in I$ (or $w \in J$ ) will be a solution of the Hopf equation up to $e^{-p}, p \in \mathbf{N}_{0}$ in the sense of $\mathbf{R}\langle\varepsilon\rangle$-distributions if for any $t \in[0, T]$

$$
\begin{gathered}
\int_{-\infty}^{+\infty}\left\{v_{t}(t, x, \varepsilon)+v(t, x, \varepsilon) v_{x}(t, x, \varepsilon)\right\} \psi(x) d x=\sum_{k=p}^{+\infty} \xi_{k} \varepsilon^{k} \in \mathbf{R}\langle\varepsilon\rangle, \\
\int_{-\infty}^{+\infty}\left\{w_{t}(t, x, \varepsilon)+w(t, x, \varepsilon) w_{x}(t, x, \varepsilon)\right\} \psi(x) d x=\sum_{k=p}^{+\infty} \eta_{k} \varepsilon^{k} \in \mathbf{R}\langle\varepsilon\rangle
\end{gathered}
$$

for every $\psi \in \mathcal{S}(\mathbf{R})$. In case when $p$ is equal to $+\infty$ the function $v(t, x, \varepsilon)$ (or $w(t, x, \varepsilon))$ exactly satisfies the Hopf equation in the sense of $\mathbf{R}\langle\varepsilon\rangle$-distributions. 
Certainly, instead of the Hopf equation one can consider some other conservation law.

From mathematical point of view, we deal with a infinitely differentiable functions in definitions 3.1 and 3.2 , so that we avoid the problem of distribution multiplication. From physical point of view, functions from the set $I$ or $J$ can describe fast processes. Mathematical models of such processes based on functions from $I$ or $J$ may give additional information and take in account a short zone where physical system make a jump from one position to another.

Thus, we will consider solutions of the Hopf equation which are infinitely narrow solitons or shock waves. It easy to see that

$$
\begin{gathered}
v(t, x, \varepsilon) \longrightarrow\left\{\begin{array}{ll}
l_{0}+\Delta l \varphi(0), & \text { if } x=c t, \\
l_{0}, & \text { if } x \neq c t .
\end{array} \text { as } \varepsilon \rightarrow 0\right. \\
w(t, x, \varepsilon) \longrightarrow h_{0}+\Delta h H(x-a t), \quad \text { as } \quad \varepsilon \rightarrow 0
\end{gathered}
$$

here $H$ is Heaviside function.

\section{NUMERICAL CALCULATION OF THE MICROSCOPIC PROFILES OF SOLITON LIKE SOLUTIONS OF THE HOPF EQUATION IN THE SENSE OF R $\langle\varepsilon\rangle$-DISTRIBUTIONS}

Thus, conservation laws are integral expressions. Therefore, it is natural, that one can interpret the Hopf equation in the sense of the definition 3.3.

We will seek a solution of the Hopf equation in the type of infinitely narrow soliton, i.e. let $v \in I$. Substituting $v(t, x, \varepsilon)$ into integral expression (3.1) and using the following formulas

$$
\begin{aligned}
& \int_{-\infty}^{+\infty} \frac{\partial}{\partial t}\left\{\varphi\left(\frac{x-c t}{\varepsilon}\right)\right\} \psi(x) d x=\sum_{k=0}^{+\infty} c \varepsilon^{k+1} m_{k} \frac{1}{k !} \psi^{(k+1)}(c t), \\
& \int_{-\infty}^{+\infty} \frac{\partial}{\partial x}\left\{\frac{1}{2} \varphi^{2}\left(\frac{x-c t}{\varepsilon}\right)\right\} \psi(x) d x=\sum_{k=0}^{+\infty}-\varepsilon^{k+1} g_{k} \frac{1}{k !} \psi^{(k+1)}(c t) .
\end{aligned}
$$

we obtain

$$
\int_{-\infty}^{+\infty}\left\{v_{t}+v v_{x}\right\} \psi d x=\sum_{k=0}^{+\infty}\left\{\Delta l\left(c-l_{0}\right) m_{k}-(\Delta l)^{2} g_{k}\right\} \varepsilon^{k+1} \frac{\psi^{(k+1)}(c t)}{k !} .
$$

Here we denoted

$$
m_{k}(\varphi)=\int_{-\infty}^{+\infty} y^{k} \varphi(y) d y, g_{k}(\varphi)=\int_{-\infty}^{+\infty} y^{k} \frac{\varphi^{2}(y)}{2} d y, k=0,1,2, \ldots
$$


From the last expression we have conditions for the function $\varphi(x)$. Namely,

$$
g_{k}(\varphi)-\frac{c-l_{0}}{\Delta l} m_{k}(\varphi)=0, \quad k \geq 0
$$

From the first $(k=0)$ equation we have

$$
\frac{c-l_{0}}{\Delta l}=\frac{g_{0}}{m_{0}}=\frac{1}{2} \int_{-\infty}^{+\infty} \varphi^{2}(x) d x
$$

Hence, we can rewrite conditions (4.2) as follows

$$
\left(\int_{-\infty}^{+\infty} \varphi^{2}(x) d x\right)\left(\int_{-\infty}^{+\infty} x^{k} \varphi(x) d x\right)=\int_{-\infty}^{+\infty} x^{k} \varphi^{2}(x) d x, \quad k \geq 0,1,2
$$

Now, let us prove the following lemma.

Lemma 4.1. For any non-negative integer $n$ there exists such function $\varphi \in$ $\mathcal{S}(\mathbf{R}), \varphi \not \equiv 0$ which satisfies the following system of non-linear equations:

$$
\int_{-\infty}^{+\infty} x^{k} \varphi(x) d x=\frac{\int_{-\infty}^{+\infty} x^{k} \varphi^{2}(x) d x}{\int_{-\infty}^{+\infty} \varphi^{2}(x) d x}, \quad k=0,1,2 \ldots n .
$$

Proof. First, we will seek function $\varphi(x)$ in the following form:

$$
\varphi(x)=c_{0} h_{0}(x)+c_{1} h_{1}(x)+\ldots+c_{n} h_{n}(x),
$$

where

$$
h_{k}(x)=\frac{H_{k}(x)}{\sqrt{2^{k} k !} \cdot \sqrt[4]{\pi}} e^{-x^{2} / 2}
$$

are Hermit functions. Then we substitute expression (4.6) into conditions (4.5). After that we will have nonlinear system of $n+1$ equations with $n+1$ unknowns $\left(c_{0}, c_{1}, c_{2}, \ldots, c_{n}\right)$. We write this system by the following way

$$
A \vec{x}=\mathcal{N}(\vec{x}), \quad \vec{x}=\left(c_{0}, c_{1}, \ldots, c_{n}\right),
$$

here $A$ is a matrix with elements

$$
A_{k j}=\int_{-\infty}^{+\infty} x^{k} h_{j}(x) d x=(-i)^{j} i^{k} \sqrt{2 \pi} h_{j}^{(k)}(0), \quad i=\sqrt{-1}, \quad k, j=0,1,2, \ldots n
$$


$\mathcal{N}$ is a nonlinear map such that

$$
\mathcal{N}(\vec{x})=\frac{1}{\|\vec{x}\|^{2}} \sum_{k=0}^{n}(N(k) \vec{x}, \vec{x}) \vec{e}_{k} \equiv \sum_{k=0}^{n} f_{k}(\vec{x}) \vec{e}_{k}
$$

Vector $\vec{e}_{k}=\left(e_{0}, e_{1}, \ldots, e_{n}\right)$ is such that $e_{k}=1$ and $e_{j}=0$ for all $j \neq k . N(k)$ are matrices with elements

$$
N_{i j}(k)=\int_{-\infty}^{+\infty} x^{k} h_{i}(x) h_{j}(x) d x, i, j, k=0,1,2 \ldots n
$$

and functions

$$
f_{k}(\vec{x})=\frac{(N(k) \vec{x}, \vec{x})}{\|\vec{x}\|^{2}} .
$$

Note that functions $f_{k}(\vec{x})$ are continuous everywhere except $\vec{x}=0$ and $\left|f_{k}(\vec{x})\right| \leq\|N(k)\|$ due to Cauchy-Bunyakovskii inequality. Matrix $A$ is invertible for any $n$ because $\operatorname{det}(A)$ is a Wronskian for the linear independent system of Hermit functions $h_{0}(x), h_{1}(x), \ldots h_{n}(x)$ and

$$
\operatorname{det}(A)=(2 \pi)^{\frac{(n+1)}{2}} W\left(h_{0}(0), h_{1}(0), \ldots h_{n}(0)\right) .
$$

We can write the system (4.7) as

$$
\vec{x}=\sum_{k=0}^{n} f_{k}(\vec{x}) A^{-1} \vec{e}_{k} \equiv F(\vec{x}) \text { or } \vec{x}=A^{-1}(\mathcal{N}(\vec{x})) \equiv F(\vec{x})
$$

Let us describe the function $F: \mathbf{R}^{n+1} \longmapsto \mathbf{R}^{n+1}$. It is continuous except at $\vec{x}=0$ and bounded. Indeed,

$$
\|F(\vec{x})\| \leq\left\|A^{-1}\right\| \sum_{k=0}^{n}\|N(k)\|, \quad r_{n}=\left\|A^{-1}\right\| \sum_{k=0}^{n}\|N(k)\| .
$$

Let us consider function $\mathcal{N}(\vec{x})$. It is continuous function everywhere in $\mathbf{R}^{n+1}$ except $\vec{x}=0$ and, moreover, $\mathcal{N}\left(\mathbf{R}^{n+1} \backslash\{0\}\right) \subset \Pi_{1}$ where $\Pi_{1}=\left\{\vec{z} \in \mathbf{R}^{n+1}\right.$ : $\left.z_{0}=1\right\}$ is a plane. Further $A^{-1}\left(\Pi_{1}\right)=\Pi_{2}$ where $\Pi_{2}=\left\{\vec{y} \in \mathbf{R}^{n+1}\right.$ : $\left.\sum_{k=0}^{n} a_{0 j} y_{j}=1\right\}$ is another plane.

$$
A_{0 j}=\int_{-\infty}^{+\infty} h_{j}(x) d x=(-i)^{j} \sqrt{2 \pi} h_{j}(0), i=\sqrt{-1}, j=0,1,2, \ldots n
$$


Thus, we can consider the function $F(\vec{x})$ which is defined on the convex compact set $C_{n}=\Pi_{2} \bigcap B\left[0, r_{n}\right]$ such that $F: C_{n} \longmapsto C_{n}$, where $B\left[0, r_{n}\right]$ is a closed ball with radius $r_{n}$. Function $F$ is continuous on the $C_{n}$ because of $\overrightarrow{0} \notin C_{n}$. Now we can use J. Schauder theorem [28].

Theorem 4.1 [Schauder fixed-point theorem]. Let $C$ be a compact convex subset of a normed space $E$. Then each continuous map $F: C \longmapsto C$ has at least one fixed point.

Hence, we can conclude that our system (4.8) and therefore system (4.7) has at least one solution. Thus, there is a function $\varphi(x)$ which satisfies to conditions (4.5) proposed lemma.

Remark 4.1. Let assume that function $\varphi(x)$ satisfies Lemma 4.1 condition. If $\beta \in \mathbf{R}$ then the function $\varphi(x+\beta)$ also satisfies this Lemma 4.1 condition. Moreover, if $\int_{-\infty}^{+\infty} \varphi^{2}(x) d x=\alpha$ then $\varphi(\alpha x)$ satisfies Lemma 4.1 condition.

Thus, we can formulate the following result.

Theorem 4.2. For any integer $p$ there is a infinitely narrow soliton type solution of the Hopf equation (in the sense of the definition 3.3) up to $e^{-p}$ with respect to the norm $|\cdot|_{\nu}$, i.e.

$$
v(t, x, \varepsilon)=l_{0}+\Delta l \varphi\left(\frac{x-c t}{\varepsilon}\right),
$$

here $l_{0}, \Delta l, c$ are real numbers, $\Delta l \neq 0$ and $\varphi \in \mathcal{S}(\mathbf{R}), \int_{-\infty}^{+\infty} \varphi(y) d y=1$. Moreover,

$$
\frac{c-l_{0}}{\Delta l}=\frac{1}{2} \int_{-\infty}^{+\infty} \varphi^{2}(x) d x
$$

For example, calculations in case $p=7$ give the "profile" $\varphi(x)$ (see Fig. 1) for the infinitely narrow soliton $v(t, x, \varepsilon)=\varphi\left(\frac{x-c t}{\varepsilon}\right)$ :

$$
\varphi(x)=\left\{\frac{c_{0}}{\sqrt[4]{\pi}}+\frac{c_{2}\left(4 x^{2}-2\right)}{\sqrt{2^{2} 2 !} \sqrt[4]{\pi}}+\frac{c_{4}\left(16 x^{4}-48 x^{2}+12\right)}{\sqrt{2^{4} 4 !} \sqrt[4]{\pi}}\right\} e^{-x^{2} / 2}
$$

where $c_{0}=0.66583, c_{2}=-0.23404, c_{4}=0.05028, c=0.25032(c$ is a velocity of the soliton). Numbers $c_{0}, c_{2}, c_{4}$ and $c$ were found approximately by iteration 
method

$$
\vec{x}_{m+1}=A^{-1}\left(\mathcal{N}\left(\vec{x}_{m}\right)\right), m \geq 0 .
$$

Matrix $A$ and a nonlinear map $\mathcal{N}$ were introduced in the proof Lemma 4.1.

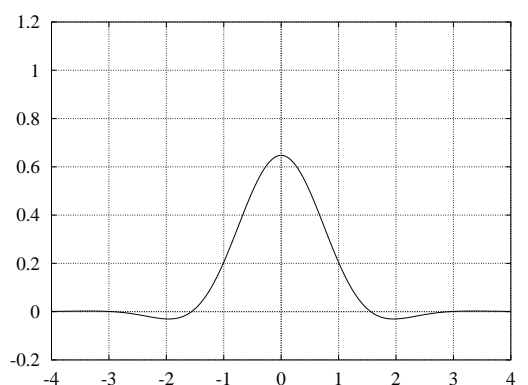

Figure 1. The case $p=7, c=0.25032$.

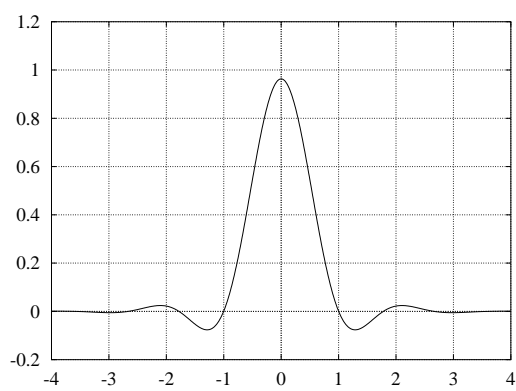

Figure 3. The case $p=15, c=0.38267$.

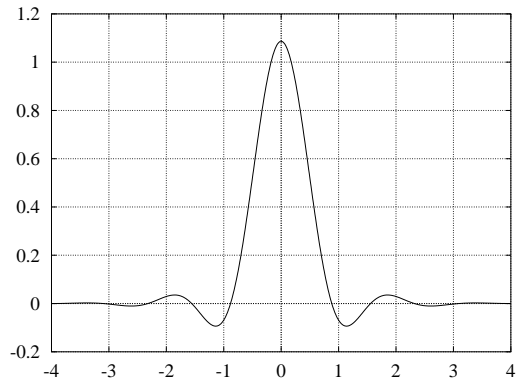

Figure 5. The case $p=19, c=0.43357$.

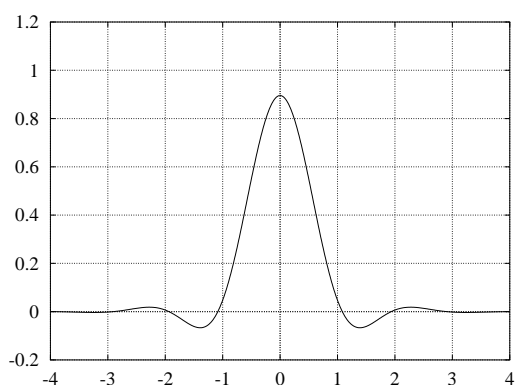

Figure 2. The case $p=13, c=0.35442$.

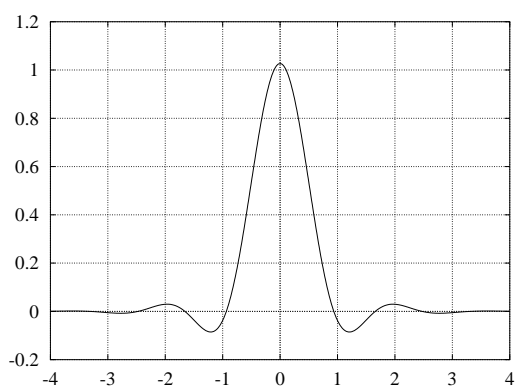

Figure 4. The case $p=17, c=0.40892$.

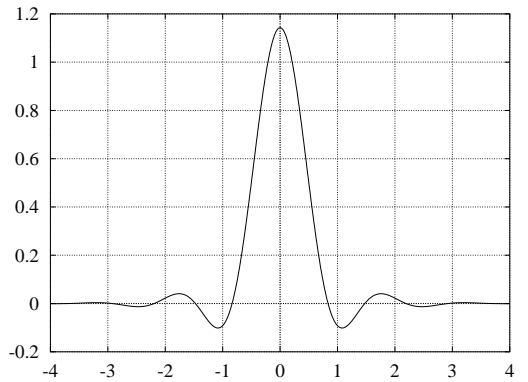

Figure 6. The case $p=21, c=0.45678$.

Soliton-like profiles $\varphi(x)$ for the Hopf equation in case $p=13,15,17,19$, 21 are given in Fig. 2, 3, 4, 5, 6 .

For the $p$ greater than 21 matrix $A$ is close to singular and calculations can be inaccurate. 


\section{CONCLUSIONS AND REMARKS}

In fact, we considered only special kind of solutions from the sets $I$ and $J$. Moreover, mentioned solutions are "approximate" solutions. It is open question about existence of the solution of the Hopf equation in sense of the definition 3.3 when $p=\infty$.

We should notice that there is also a Non-Archimedean approach which is developed by V. Vladimirov, I. Volovich, E. Zelenov [32], A. Khrennikov [13]. This approach is based on $p$-adic valued distributions and is used for the construction of some models in Mathematical Physics.

The authors of the papers $[4 ; 7]$ considered the same equations but they speculated a different ideology for generalized solutions and generalized functions.

\section{Acknowledgment}

It is pleasure to thank the seminar of Moscow Energy Institute organized by Prof. Yu.A. Dubinskii for the consideration to this work.

Research is partially supported by Belarussian Fundamental Research Foundation Grant No F99M-082.

\section{REFERENCES}

[1] A.B. Antonevich, Ya.V. Radyno and N.Ya. Radyno. About the theory of mnemofunctions and its applications. Integral Transforms and Special Functions, 6(1-4), 123 134, 1998.

[2] H.A. Biagioni. A nonlinear theory of generalized functions. Lecture Notes in Math., 1421, 1990.

[3] J.-F. Colombeau. Elementary Introduction to New Generalized Functions. NorthHolland, Amsterdam, 1985.

[4] J.-F. Colombeau, A.Y. Le Roux, A. Noussair and B. Perrot. Microscopic profiles of shock waves and ambiguities in multiplications of distributions. SIAM J. Numer. Anal., 26(4), $871-883,1989$.

[5] V.G. Danilov, V.P. Maslov and V.M. Shelkovich. Algebras of singularities of singular solutions to the first-order quasi linear strictly hyperbolic systems. Theor. and Math. Physics, 114(1), 3 - 55, 1998.

[6] A. Delcroix and D. Scarpalezos. Asymptotic scales-asymptotic algebras. Integral Transforms and Special Functions, 6(1-4), 157 - 166, 1997.

[7] Yu.V. Egorov. On the theory of generalized functions. Usp. Mat. Nauk, 45, 3 - 40, 1990.

[8] J. Goodman and P. Lax. On dispersive difference schemes I. Comm. Pure and Appl. Math., 41, 591 - 613, 1988.

[9] E. Hopf. The partial differential equation $u_{t}+u u_{x}=\mu u_{x x}$. Comm. Pure Appl. Math., 3, $201-230,1950$. 
[10] T.Y. Hou and P. Lax. Dispersive Approximation in Fluid Dynamics. Comm. Pure and Appl. Math., XLIV, 1 - 40, 1991.

[11] L.V. Kantorovich and G.P. Akilov. Functional analysis in the normed spaces. Fizmatgiz, Moscow, 1957. (In Russian)

[12] B. Keyfitz. Conservation Laws, Delta-Shocks and Singular Shocks. In: Nonlinear Theory of Generalized Functions, volume 401 of Research Notes in Mathematics, Chapman and Hall, London, 99 - 111, 1999.

[13] A. Khrennikov. p-adic Valued Distributions in Mathematical Physics. Kluwer Academic Publishers, 1994.

[14] P. Lax. On dispersive difference schemes. Physica 18D, 250 - 254, 1986.

[15] P. Lax. The Zero Dispersion Limit, a Deterministic Analogue of Turbulence. Comm. Pure and Appl. Math., XLIV, 1047 - 1056, 1991.

[16] A. Lightstone and A. Robinson. Nonarchimedian Fields and Asymptotic Expantions. North-Holland Publishing Company, Amsterdam, 1975.

[17] J.-A. Marti. $(\mathcal{C}, \mathcal{E}, \mathcal{P})$-Sheaf Structures and Applications. In: Nonlinear Theory of Generalized Functions, volume 401 of Research Notes in Mathematics, Chapman and Hall, London, 175 - 186, 1999.

[18] V.P. Maslov and G.A. Omel'yanov. Asymptotic soliton-form solutions of equations with small dispersion. Uspekhi Mat. Nauk, 36(3), 63 - 126, 1981.

[19] M. Nedeljkov, S. Pilipović and D. Scarpalézos. The Linear Theory of Colombeau Generalized Functions, volume 385. Longman, 1998.

[20] J. Von Neumann. Proposal and Anylysis of a Numerical Method for the Treatment of Hydrodymamical Shock Problem, volume VI. Collected Works, Pergamon, London, 1963.

[21] J. Von Neumann and R.D. Richtmyer. Method for the Numerical Calculation of Hydrodynamic Shocks. J. Appl. Phys., 21, $232-237,1950$.

[22] M. Oberguggenberge. Multiplication of distributions and applications to partial differential equations. Pitman Research Notes Math. Series, 259, 1992.

[23] O.A. Oleinik. Construction of a generalized solution of the Cauchy problem for a quasilinear equation of the first order by introduction of "vanishing viscosity". Uspekhi Mat. Nauk., 14(2), 160 - 164, 1959.

[24] M. Radyna. Functionals with values in the Non-Archimedean field of Laurent series and Method for Numerical Calculations of Shocks and Soliton like Solutions of Some Conservation Laws.

[25] N.Ya. Radyno. New construction of generalized functions and its application to the Hopf equation. Proceedings of National Academy of Sciences of Belarus, 3, 2000.

[26] N.Ya. Radyno. Calculation method for some solutions of the Hopf equation and generalization of Sobolev-Schwartz distributions. Lietuvos matematikos rinkinys, $\mathbf{4 1 ( 1 )}, 93$ - 107, 2001. In Russian)

[27] E.E. Rosinger. Generalized solutions of nonlinear partial differential equations, volume 146. North Holland Math. Studies, Amsterdam, 1987.

[28] J. Schauder. Der Fixpunktsatz in Funktionalröumen. Stadia Math., 2, 171 - 180, 1930.

[29] L. Schwartz. Theorie des distributions. Part 1; Part 2. 1951.

[30] S.L. Sobolev. Méthode nouvelle à resoudre le problème de Cauchy pour les équations linéaires hyperboliques normales. Mat. Sb., 43(1), 39 - 71, 1936.

[31] T. Todorov. Pointwise Values and Fundamental Theorem in the Algebra of Asymptotic Functions. In: Nonlinear Theory of Generalized Functions, volume 401 of Research Notes in Mathematics, London, 369 - 383, 1999. 
[32] V. Vladimirov, I. Volovich and E. Zelenov. p-adic analysis and mathematical physics. Nauka, Moscow, 1994.

Funkcionalai su reikšmėmis ne-Archmediniuose Laurent'o sekų laukuose ir jų taikymai elastiškumo teorijos lygtims

M. Radyna

Funkcionalai su reikšmėmis ne-archimediniuose Laurent'o sekų laukuose pritaikyti apibrež̌ti apibendrintaji Hop'o lygties sprendinị solitono pavidalu. Pasiūlytas skaitinis algoritmas begalo siauro solitono profilio radimui. Taikant ši metodą, profilio radimas suvedamas i netiesinès algebrinių lygčių sistemos erdveje $R^{n+1}, n>1$, sprendimą. Parodyta, kad kai kuriuos sprendinius galima surasti naudojant Niutono iteracini metodą. Pateikiami pavyzdžiai ir skaitiniai testai. 